\title{
Urgência e emergência em tempos de COVID-19 - uma revisão integrativa da
}

\section{literatura}

\author{
Urgency and emergency in times of COVID-19 - an integrative literature review \\ Urgencia y emergencia durante COVID-19 - una revisión integrativa de la literatura
}

Regina Consolação dos Santos ORCID: https://orcid.org/0000-0002-7393-7210 Universidade de Itaúna, Brasil

E-mail: reginasantos72@outlook.com

Thais Andrade Carvalho

ORCID: https://orcid.org/0000-0002-0043-3725

Universidade de Itaúna, Brasil

E-mail: thaiscarvalho-@outlook.com

Isnard Fernandes Souza Neto

ORCID: https://orcid.org/0000-0002-9663-2816

Universidade de Itaúna, Brasil

E-mail: isnardfernandes14@gmail.com

Thayane Vieira Carvalho

ORCID: https://orcid.org/0000-0001-8644-8954

Clínica Auge, Brasil

E-mail: thayanevieira100@yahoo.com.br

Thays Lorena Bahia Vieira Correia

ORCID: https://orcid.org/0000-0001-8308-8746

Universidade de Itaúna, Brasil

E-mail: lorenathays27@gmail.com

Silmara Nunes Andrade

ORCID: https://orcid.org/0000-0002-1975-0827 Universidade do Estado de Minas Gerais, Brasil

E-mail: silmara.andrade@uemg.br

Isabela Camargos Guimarães

ORCID: https://orcid.org/0000-0002-0155-2750

Universidade de Itaúna, Brasil

E-mail: isabelacamargosg@gmail.com

Thays Cristina Pereira Barbosa

ORCID: https://orcid.org/0000-0003-1816-0662 Universidade do Estado de Minas Gerais, Brasil

E-mail: thayscristina19@gmail.com

Claudia Martins da Costa

ORCID: https://orcid.org/0000-0001-5904-6829 Universidade do Estado de Minas Gerais, Brasil

E-mail: claudiacostamello.92@gmail.com

Heber Paulino Pena

ORCID: https://orcid.org/0000-0002-9122-6827

Universidade de Itaúna, Brasil

E-mail: heberppena@yahoo.com.br

\begin{abstract}
Resumo
O presente estudo tem como objetivo analisar as mudanças ocorridas no atendimento hospitalar de urgência e emergência durante a pandemia do COVID-19 e elaboração de estratégias para prevenir os riscos de colapso dos serviços de saúde. Trata-se de uma revisão integrativa da literatura sendo pesquisados estudos científicos entre os meses de setembro e outubro de 2020, nas bases de dados PubMed, Biblioteca Virtual em Saúde do Ministério da Saúde e Google Acadêmico, com os descritores "atendimento", "emergência", "COVID-19" e "pandemia". Foram encontrados 689 artigos nas três bases de dados sendo selecionados 28 estudos que abordavam a temática discutida, para elaboração da revisão sendo eles em português inglês e espanhol. Os estudos trouxeram informações sobre as modificações epidemiológicas nos departamentos de urgência e emergência, evidenciando um novo perfil de atendimento, mostrando a forma e estratégias que os epicentros da pandemia no mundo e o Brasil se organizaram. Destaca-se que através da literatura cientifica foi possível identificar as principais mudanças ocorridas no atendimento de urgência e emergência, além de adaptações nos setores hospitalares e na criação de novos ambulatórios para diminuir a sobrecarga dos serviços de saúde, consequentemente, evitando o seu colapso.
\end{abstract}


Palavras-chave: Atendimento de emergência; COVID-19; Pandemia.

\begin{abstract}
This study aims to analyze the changes that occurred in urgent and emergency hospital care during the COVID-19 pandemic and to develop strategies to prevent the risks of health services collapse. This is an integrative review of the literature, with scientific studies being searched between the months of September and October 2020, in the databases PubMed, Virtual Health Library of the Ministry of Health and Google Scholar, with the descriptors "attendance", "emergency", "COVID-19" and "pandemic". 689 articles were found in the three databases and 28 studies were selected that addressed the topic discussed, for the preparation of the review, in English, Portuguese and Spanish. The studies brought information about the epidemiological changes in the urgency and emergency departments, showing a new profile of care, showing the form and strategies that the epicenters of the pandemic in the world and Brazil have organized. It is noteworthy that through the scientific literature it was possible to identify the main changes that occurred in urgent and emergency care, in addition to adaptations in the hospital sectors and in the creation of new outpatient clinics to reduce the overload of health services, consequently, preventing their collapse.
\end{abstract}

Keywords: Emergency care; COVID-19; Pandemic.

\title{
Resumen
}

This study aims to analyze the changes that occurred in urgent and emergency hospital care during the COVID-19 pandemic and to develop strategies to prevent the risks of health services collapse. This is an integrative review of the literature, with scientific studies being searched between the months of September and October 2020, in the databases PubMed, Virtual Health Library of the Ministry of Health and Google Scholar, with the descriptors "attendance", "emergency", "COVID-19" and "pandemic". 689 articles were found in the three databases and 28 studies were selected that addressed the topic discussed, for the preparation of the review, in English, Portuguese and Spanish. The studies brought information about the epidemiological changes in the urgency and emergency departments, showing a new profile of care, showing the form and strategies that the epicenters of the pandemic in the world and Brazil have organized. It is noteworthy that through the scientific literature it was possible to identify the main changes that occurred in urgent and emergency care, in addition to adaptations in the hospital sectors and in the creation of new outpatient clinics to reduce the overload of health services, consequently, preventing their collapse.

Palabras clave: Cuidados de emergencia; COVID-19; Pandemia.

\section{Introdução}

O vírus Severe Acute Respiratory Syndrome, Coronavirus 2 (SARS-CoV-2), ficou conhecido mundialmente como COVID-19 e foi o responsável pelo aparecimento expressivo de casos de pneumonia viral em todo o mundo. Os primeiros registros da doença ocorreram na cidade de Wuhan, capital da província de Hubei, na China, em dezembro de 2019 e, dois meses depois, em fevereiro de 2020, foi registrado o primeiro caso de COVID-19 em território brasileiro, na cidade de São Paulo (Iser et al., 2020; Mazon, Komuchena, Roik, Wieczorkievicz \& Ditterich, 2020).

Em 11 de março de 2020, a Organização Mundial da Saúde (OMS) caracterizou a COVID-19 como uma pandemia, termo utilizado para indicar que uma epidemia se espalhou para mais de dois continentes, como ocorreu com o SARS-CoV-2. $\mathrm{O}$ vírus se proliferou rapidamente por todo o mundo devido a sua alta transmissibilidade $\mathrm{e}$, atualmente, apresenta um número superior a 93.000 .000 infectados e 2.000.000 mortes, dados que sofrem mudanças diariamente, devido ao contágio permanecer em ascensão (Brasil, 2020; Who, 2020).

A COVID-19, pertencente à família Coronaviridae do grupo das Coronaviruses (CoVs), é uma doença caracterizada por causar comprometimento pulmonar nos pacientes e pode apresentar manifestações clínicas diversas, das mais leves as mais graves, promovendo sinais e sintomas distintos de pessoa para pessoa, após contágio por gotículas e/ou contato (Iser et al., 2020). Segundo o Ministério da Saúde, dentre os sintomas, destacam-se as síndromes Gripais (SG), onde os pacientes apresentam tosse, febre, dor de garganta, cefaleia, mialgia, entre outros; e a Síndrome Respiratória Aguda Grave (SRAG), se manifestando com febre, acompanhada de tosse ou dor de garganta com presença de dispneia e hospitalização; além de se manifestar também com sintomas como hipoxemia, taquipnéia e hipotensão (Brasil, 2020).

A alta demanda implica na necessidade de racionar equipamentos e profissionais da saúde, exigindo dos hospitais um preparo assíduo para expandir e remanejar a assistência de forma planejada, elaborando protocolos de atendimentos alinhados 
ao plano de contingência estadual e federal para nortear os atendimentos de casos suspeitos e confirmados, além de proporcionar aos profissionais, capacitação e fluxos de atendimento adequados, entre outros (Engstrom, Melo, Giovanella, Mendes, Grabois \& Mendonça, 2020).

Diante da necessidade de garantir que as pessoas recebam atendimento de qualidade, chegou-se à construção da pergunta norteadora desta pesquisa: $\mathrm{O}$ que as pesquisas científicas e investigações em saúde vêm apontando sobre os atendimentos de urgência e emergência hospitalar durante a pandemia da COVID-19?

Investigar e discutir a questão sobre o que as pesquisas científicas em saúde vêm apontando no que diz respeito aos atendimentos de urgência e emergência hospitalar durante a pandemia da COVID-19 permite visualizar o que vem sendo feito e quais são as lacunas existentes, no que tange aos aspectos de prevenção à sobrecarga e/ou colapso dos sistemas de saúde. Essa identificação da produção acadêmica e investigações em saúde permite contribuir com discussões sobre possibilidades de atendimento hospitalar para prevenção no colapso do sistema de saúde em vista da alta proliferação viral trazida pela pandemia.

Assim, a importância dessa pesquisa se dá em vista da necessidade de se lidar com as demandas urgentes e especificas da COVID-19, principalmente no que se relaciona a internação e garantia de qualidade de atendimento, saúde e vida dos pacientes. O presente trabalho teve como objetivo analisar as mudanças ocorridas no atendimento hospitalar de urgência e emergência durante a pandemia da COVID-19 e elaboração de estratégias para prevenir os riscos de colapso dos serviços de saúde através de uma revisão integrativa da literatura.

\section{Metodologia}

O estudo consistiu em uma revisão integrativa da literatura, de caráter descritivo e análise qualitativa, cuja abordagem foi exploratória com interpretação dos dados coletados, norteados por questões elaboradas no propósito de discutir sobre as situações e mudanças ocorridas nos serviços de urgência e emergência hospitalar durante o período pandêmico, causado pelo SARS-CoV-2 (Souza, Silva \& Carvalho, 2010; Pereira, Shitsuka, Parreira \& Shitsuka, 2018).

Realizou-se uma busca por artigos nos períodos de setembro e outubro de 2020 na seguinte base de dados: Pubmed, Biblioteca Virtual em Saúde do Ministério da Saúde e Google acadêmico. Foram utilizados, para realizar a pesquisa, os seguintes descritores: "atendimento", "emergência", “COVID-19" e "pandemia”, e os termos Mesh: "emergencies", "COVID-

19", "emergency tratment", "pandemics", sendo inseridos os operadores booleanos $A N D$, e $O R$, que ofereceram as intercessões entre os descritores de pesquisa escolhidos, bem como possibilidades.

Os critérios de inclusão para elaboração da revisão foram: Artigos que abordavam as formas de atendimento de urgência e emergência durante a pandemia, artigos em português, inglês e espanhol. Foram excluídos os artigos que fugiam da temática abordada, artigos incompletos, e artigos que tratavam aspectos patológicos da COVID-19.

\section{Resultados e Discussão}

Foi identificada na literatura científica e investigações em saúde por meio de uma revisão integrativa nas bases de dados Pubmed, Biblioteca Virtual em Saúde do Ministério da Saúde e Google acadêmico, momento no qual se apresentou os achados e seus aspectos quantitativos que evidenciam situações que podem ou tem gerado problemas no sistema de saúde brasileiro. Visto isso, a seguir será demonstrado o processo de busca dos artigos (Figura 1). 
Figura 1. Fluxograma referente à busca de artigos, seguido as recomendações PRISMA.

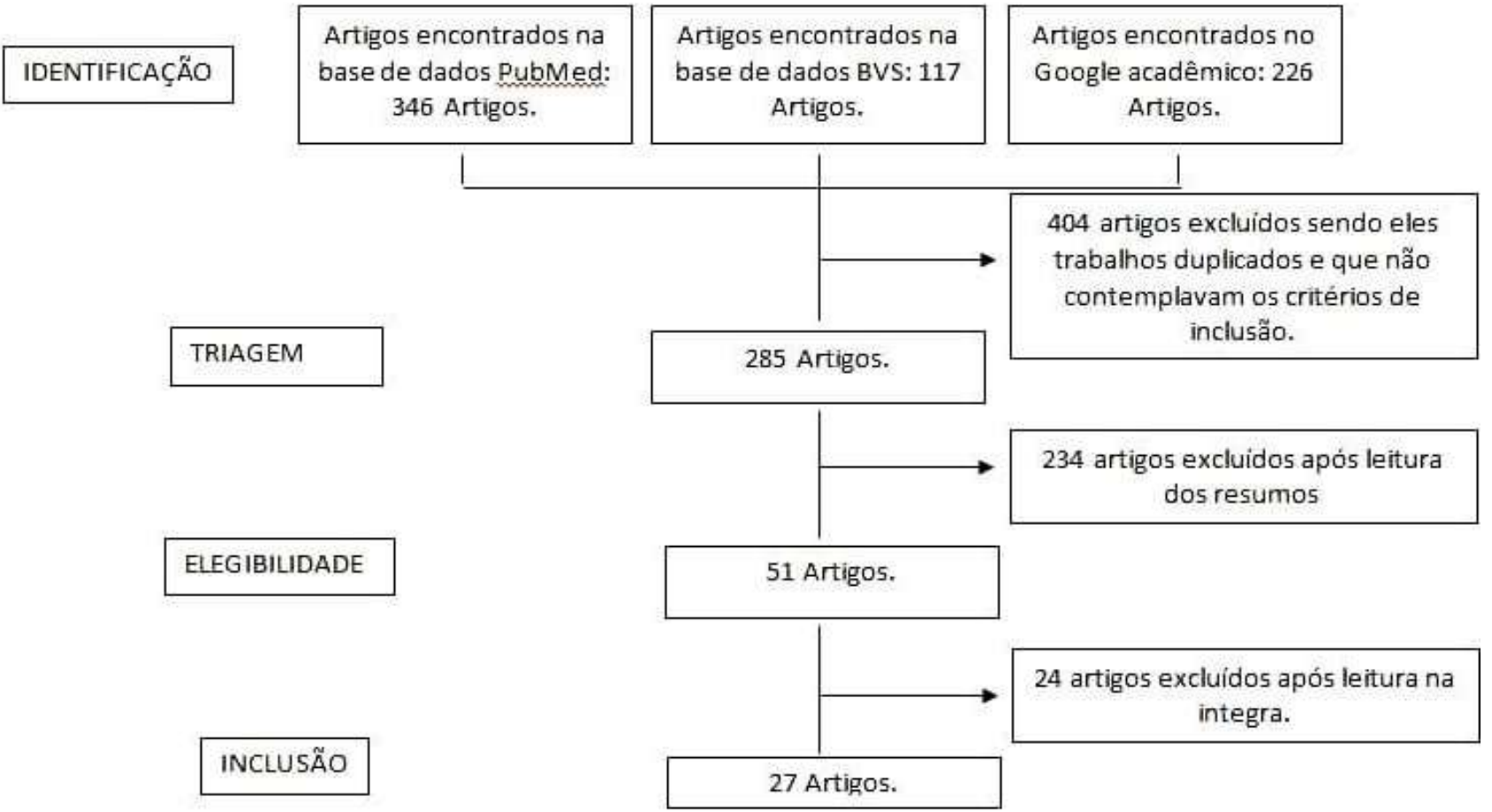

Fonte: Acervo Pessoal Dos Autores. Imagem Autorizada.

Sendo assim, foram encontrados ao total 689 artigos nas três bases de dados selecionadas, restando 285 artigos depois de realizados os critérios de exclusão e inclusão, 51 artigos após a avaliação dos resumos e 27 artigos lidos na íntegra para elaboração da revisão. Após isso, os artigos lidos na íntegra foram analisados e listados na Tabela 1.

Tabela 1. Artigos utilizados na revisão destacando seus autores, ano e local de publicação.

\begin{tabular}{lll}
\hline Autor/ano de publicação & Título Local de publicação
\end{tabular}

$\begin{array}{ll}\text { Farias et al./2020 } & \begin{array}{l}\text { O papel da atenção primária no combate ao COVID-19: } \\ \text { impacto na saúde pública e perspectivas futuras }\end{array}\end{array}$

Ferreira L. et al./2020 Coronavirus - O que fazer hoje, olhando o amanhã? $\quad$ Associação Médica Brasileira.

Engstro E. et al./2020 Recomendações para a organização da Atenção Observatório COVID-19 Fiocruz. Primária à Saúde no SUS no enfrentamento da COVID19.

\begin{tabular}{llllll}
\hline $\begin{array}{l}\text { Schuchmann } \\
\text { al./2020 }\end{array}$ & AZ. et & $\begin{array}{l}\text { Dilemas sanitários e sociais no enfrentamento da } \\
\text { pandemia de COVID-19 / Isolamento social vertical X } \\
\text { Isolamento social horizontal: saúde e dilemas sociais no } \\
\text { enfrentamento da pandemia COVID-19. }\end{array}$ & Revista Brasileira de Revisão de Sa \\
\hline Silva LLS. et al./2020 & $\begin{array}{l}\text { Medidas de distanciamento social para o enfrentamento } \\
\text { da COVID-19 no Brasil: caracterização e análise } \\
\text { epidemiológica por estado }\end{array}$ & Caderno de Saúde Pública. \\
\hline $\begin{array}{l}\text { Lourenção } \\
\text { al./2020 }\end{array}$ & LG. et & $\begin{array}{l}\text { A COVID-19 e os desafios para o sistema e os } \\
\text { profissionais de saúde }\end{array}$ & Revista Enfermagem em Foco.
\end{tabular}




\begin{tabular}{llll}
\hline $\begin{array}{l}\text { Barretoti } \\
\text { al./2020 }\end{array}$ & et & $\begin{array}{l}\text { O papel da atenção primária no combate ao COVID-19: } \\
\text { impacto na saúde pública e perspectivas futuras }\end{array}$ & $\begin{array}{l}\text { Revista Brasileira de Medicina de Família e } \\
\text { Comunidade. }\end{array}$ \\
\hline Cabral E. et al./2020 & $\begin{array}{l}\text { Contribuições e desafios dos Cuidados de Saúde } \\
\text { Primários em toda a pandemia COVID-19 }\end{array}$ & Revista Enfermagem em Foco. \\
\hline Santana R. et al./2020 & $\begin{array}{l}\text { A procura de serviços de urgência/emergência } \\
\text { hospitalar: tendências durante o primeiro mês de } \\
\text { resposta à COVID-19 }\end{array}$ & Escola Nacional de Saúde Pública. \\
\hline
\end{tabular}

Yao, W, et al./2020 Emergency tracheal intubation in 202 patients with COVID-19 in Wuhan, China: lessons learnt and

British Journal of Anaesthesia. international expert recommendations.

\begin{tabular}{lll}
\hline $\begin{array}{l}\text { Cervino, G., \& Oteri, } \\
\text { G./2020 }\end{array}$ & $\begin{array}{l}\text { Pandemic and Telephone Triage before Attending } \\
\text { Medical Office: Problem or Opportunity? }\end{array}$ & Medicina (Kaunas, Lithuania). \\
\hline $\begin{array}{l}\text { Lerner, E. B., Newgard, } \\
\text { C. D., \& Mann, N. }\end{array}$ & $\begin{array}{l}\text { System: A Preliminary Report. Academic emergency } \\
\text { medicine }\end{array}$ & $\begin{array}{l}\text { Official Journal of the Society for Academic } \\
\text { C./2020 }\end{array}$
\end{tabular}
C. $/ 2020$ medicine

Leow, S. H. et al./2020 The Attend Study: A Retrospective Observational Study of Emergency Department Attendances During the Early Stages of the COVID-19 Pandemic

O'Leary, F. et al./2020 Personal protective equipment in the paediatric emergency department during the COVID-19 pandemic: Estimating requirements based on staff numbers and patient presentations.

Wang, J. et al./2020

Identifying the effects of an upgraded 'fever clinic' on COVID-19 control and the workload of emergency department: retrospective study in a tertiary hospital in China.

$\begin{aligned} & \text { Mitchell, } \\ & \text { al./2020 }\end{aligned}$

Blackhall, K. K. et Provision of Emergency Maxillofacial Service During
al./2020 the COVID-19 Pandemic: A Collaborative Five Centre UK Study.

\section{Cureus.}

Emergency Medicine Australasia : EMA.

BMJ

Emergency Medicine Australasia : EMA

The British Journal of Oral \& Maxillofacial Surgery.

Disappearing act: COVID-19 and paediatric emergency

Archives of Disease in Childhood.

Dann, L.et al./2020 department attendances.

Hughes, H. et al./2020 Emergency department use during COVID-19 as Emergency Medicine Jornal. described by syndromic surveilla nce.

Lin, C. et al./2020

A Double Triage and Telemedicine Protocol to

Journal of Medical Internet Research.

Optimize Infection Control in an Emergency Department in Taiwan During the COVID-19 Pandemic.

McDonnell, T.et COVID-19:Emergency department attendance by
al./2020 paediatric patients during COVID-19 - project protocol.

Mulholland, R. H. et Impact of COVID-19 on accident and emergency al./2020 attendances and emergency and planned hospital admissions in Scotland: an interrupted time-series analysis.

Low, T. Y., Mathews,

I., Lau, J. W., \& Ngiam, Close air support: enhancing emergency care in the COVID-19 pandemic.

HRB open research.

Journal of the Royal Society of Medicine.

K. Y. $/ 2020$ 


\begin{tabular}{|c|c|c|}
\hline $\begin{array}{l}\text { Azizoddin, D. R., Vella } \\
\text { Gray, K., Dundin, A., \& } \\
\text { Szyld, D. } / 2020\end{array}$ & $\begin{array}{l}\text { Bolstering clinician resilience through an } \\
\text { interprofessional, web-based nightly debriefing } \\
\text { program for emergency departments during the } \\
\text { COVID-19 pandemic. }\end{array}$ & Journal of Interprofessional care. \\
\hline $\begin{array}{l}\text { McDonnell, T. et } \\
\text { al. } / 2020\end{array}$ & $\begin{array}{l}\text { Assessing the Impact of COVID-19 Public Health } \\
\text { Stages on Paediatric Emergency Attendance. }\end{array}$ & $\begin{array}{l}\text { International Journal of Environmental Research } \\
\text { and Public Health }\end{array}$ \\
\hline $\begin{array}{lcc}\text { Schrading, } & \text { W. } & \text { A., } \\
\text { Pigott, } & \text { D., } & \& \\
\text { Thompson, L. } / 2020 & \end{array}$ & $\begin{array}{l}\text { Virtual Remote Attending Supervision in an Academic } \\
\text { Emergency Department During the COVID-19 } \\
\text { Pandemic. }\end{array}$ & AEM education and training. \\
\hline $\begin{array}{l}\text { Mulholland, R. H. et } \\
\text { al. } / 2020\end{array}$ & $\begin{array}{l}\text { Impact of COVID-19 on accident and emergency } \\
\text { attendances and emergency and planned hospital } \\
\text { admissions in Scotland: an interrupted time-series } \\
\text { analysis. }\end{array}$ & Journal of the Royal Society of Medicine \\
\hline
\end{tabular}

Fonte: Acervo Pessoal dos autores.

Os artigos selecionados foram analisados e organizados em duas categorias temáticas, sendo: Escopo de Atendimento no Mundo e Escopo de Atendimento no Brasil.

\section{Escopo de atendimento no mundo}

Os resultados demonstram que o serviço de urgência e emergência em todo o mundo passou por muitas modificações em seu atendimento, o que fez com que esses serviços buscassem respostas com o objetivo de solucionar a crise emergencial que a pandemia da COVID-19 provocou, exigindo a criação de estratégias para suprir um novo perfil de atendimento nos epicentros (Leow, Dean, MacDonald-Nethercott, MacDonald-Nethercott \& Boyle, 2020; Yao, Wang, Jiang, Fleisher \& Wei, 2020). A primeira mudança dentro dos serviços de atendimento de urgência e emergência foi epidemiológica, o número de atendimento diários caiu em comparação com o mesmo período nos anos anteriores (Dann, Fitzsimons, Gorman, Hourihame \& Okafor, 2020).

As principais causas dessa mudança são as doenças que se apresentavam no departamento sem requerer atendimento de urgência e a redução do estresse escolar, ambas, consequências das medidas governamentais de isolamento social (Blackhall et al.,2020). No Reino Unido, esse perfil epidemiológico também se repetiu modificando seu perfil de atendimento, ocorrendo um aumento nos casos de acidentes domésticos e violência doméstica e uma redução nos casos de traumas e fraturas graves, mostrando que a taxa geral caiu nos atendimentos maxilofaciais nos departamentos de urgência e emergência (Mitchell et al., 2020; McDonnel, 2020).

A segunda modificação se deu no âmbito da triagem, realizada com o objetivo de tentar restringir o contato dos pacientes dentro do ambiente de atendimento, visto que esse contato passou a ser considerado área de foco para transmissão da COVID-19 (Lin et al., 2020). Na China, a eficácia de uma dupla triagem dentro de um pronto atendimento unido à criação de uma clínica de febre, resultou em uma redução do número de infecções cruzadas e uma redução da permanência de possíveis casos de pacientes infectados no pronto atendimento (Wang et al., 2020).

Outra grande estratégia utilizada foi a telemedicina associada à dupla triagem, que reduziu o contato do médico com os pacientes menos graves de COVID-19, diminuindo o número de contaminações e a sobrecarga dos profissionais de saúde que atuam em hospitais de urgência e emergência (Cervino \& Oteri, 2020; Azizoddin, Gray, Szyld, 2020).

Os recursos dentro de um departamento de urgência e emergência também foram observados e sofreram modificações. O uso de EPI's apresentou um aumento considerável já previsto durante a pandemia e ressalta que realocar esses 
recursos sazonalmente é de grande importância para o funcionamento dos departamentos de urgência e emergência de todas as áreas hospitalares (O'Leary, Pobre, Mariano, Tan \& Jani, 2020).

Do ponto de vista das estratégias que foram executadas pelos departamentos de urgência e emergência, houve a criação de tendas ao ar livre para dispersar o fluxo de pessoas e, consequentemente, reduzir o atendimento no departamento de urgências e emergências para evitar o risco de contaminação (Schrading, Pigot \& Tompson, 2020). Embora os resultados fossem abaixo do esperado, houve uma queda considerável no número de atendimentos nos prontos socorros (Hughes et al., 2020). Nos EUA, foi analisado a proporção do uso do 911 no período de surto da pandemia da COVID-19, onde mostrou uma redução no número de chamadas recebidas e duplicou o número de mortes do mesmo serviço. Essa estratégia unida à utilização de suporte aéreo nos departamentos de urgência e emergência resultou em uma melhora no atendimento local (Lerner, Newgard \& Mann, 2020; Low, Mathews, Liang- Lau \& Ngiam, 2020).

A diversidade dos serviços de atendimento de urgência e emergência e a forma com que cada país lida com a pandemia da COVID-19 são pontos fracos e que dificultam a tentativa de criar uma visão única de como as estruturas de urgência e emergência se organizam durante a pandemia como visto na discussão.

\section{Escopo de atendimento no Brasil}

A partir dos trabalhos analisados, percebe-se que no Brasil, o crescente número de contágio se tornou um problema que influenciou diretamente na qualidade dos atendimentos prestados pelos profissionais de saúde (Loureção, 2020). Isso se dá devido a esses profissionais ficarem expostos a longas jornadas de trabalho, falta de equipamentos de proteção e a queda do número de funcionários (Carvalho, Elias \& Carvalho, 2020).

As publicações atenderam as necessidades de dados sobre relatos referentes ao trabalho dos profissionais da saúde que atuam na linha de frente do combate à pandemia (Schuchmann et al., 2020). Nesses relatos é exposta uma situação preocupante, o comprometimento da estrutura organizacional dos hospitais, principalmente nos setores de UTIs, devido à reorganização forçada pela pandemia (Farias, Colares, Barreto \& Calvacanti, 2020).

O Brasil tem lidado com as consequências da COVID-19, para evitar a superlotação de pacientes do SUS, criando os hospitais de campanha, específicos para o atendimento de pacientes com COVID-19, o que auxilia a evitar um colapso dos tradicionais hospitais, pois atendem um número expressivo de pacientes e contribui para evitar possíveis contaminações, funcionando em colaboração com o SUS (Engstrom et al., 2020).

O que se nota nos artigos analisados é que apenas os aspectos de prevenção são abordados (Filho Silva, et al., 2020; Mitchell et al., 2020). Do ponto de vista integrativo, os trabalhos abordados têm uma conexão linear, sendo os primeiros a abordagem de previsões de medidas preventivas (Santana, Sousa, Soares, Boto \& Rocha, 2020). Por último, o relato dos profissionais da saúde traz uma caracterização da situação do trabalho nos hospitais, abordando como acontece o atendimento dos pacientes nos setores de urgência e emergência hospitalar (Who, 2020).

A falta de estudos direcionados ao setor de atendimentos de urgência e emergência hospitalar gera uma dificuldade em criar possíveis melhorias para esse modelo de atendimento, além de dificultar possíveis prevenções de sobrecargas e colapso que o sistema de saúde brasileiro pode enfrentar, considerando que o Brasil é um país de extensão continental que atende um número elevado de pessoas (Cabral et al., 2020). Por outro lado, o perfil epidemiológico encontrado em outros países também foi relatado pela Associação Médica Brasileira (AMB), que indicou uma redução drástica nos atendimentos a traumas e fraturas graves, ocasionado pelo isolamento social adotado como medida de prevenção (Amb, 2020). 


\section{Considerações Finais}

Considera-se que os estudos encontrados retratavam as modificações ocorridas no âmbito dos serviços de saúde em urgência e emergência de modo que as estratégias diversas, dentro da atenção ao cliente, forneçam melhorias no atendimento ao usuário e evita a disseminação do vírus e colapso nos hospitais. Notou-se também a diminuição de consultas e atendimentos voltados para os traumas.

No Brasil, identificou uma escassez de estudos, retratando principalmente as questões que norteavam o possível colapso no SUS. Os departamentos de urgência e emergência precisam estar atentos para criar as estratégias e formas de atender o novo perfil que foi criado durante a pandemia indo de encontro com a prevenção do contágio dos profissionais e pacientes, por meio de protocolos, articulando recursos para que não ocorra um colapso organizacional, analisando os recursos disponíveis e as políticas públicas adotadas em cada região.

Notou-se que, até o presente momento, há um número reduzido de artigos sobre o assunto. Mesmo as publicações que tratam sobre a problemática apresentaram poucas informações sobre estudos realizados neste campo, a saber, da atuação dos profissionais nos setores de atendimento de urgência e emergência. As publicações analisadas se limitam a indicar previsões, medidas preventivas e trazer dados de outros países.

Como trabalhos futuros, espera-se desenvolver estudos mais detalhados sobre a COVD-19, sobre o papel de cada profissional de saúde diante disso e o impacto causado na assistência prestada nos hospitais e pronto socorros de todo o mundo, devido a ser um tema recente e se tratar de um problema de saúde pública a nível mundial.

\section{Referências}

Associação Médica Brasileira (AMB). (2020). Diretrizes AMB: COVID-19. https://amb.org.br/diretrizes-amb-covid-19/

Azizoddin, D. R., Gray, K. V., \& Szyld, D. (2020). Bolstering clinician resilience through an interprofessional, web-based nightly debriefing program for emergency departments during the COVID-19 pandemic. Journal of interprofessional care, 34(5), 711-715. https://doi.org/10.1080/13561820.2020.1813697

Barretoti, F. K. A., Colares, M. P., Barreto, F. L. de. A., \& Calvacante, L. P. de. G. (2020). O papel da atenção primária no combate ao COVID-19: impacto na saúde pública e perspectivas futuras. Revista Brasileira de Medicina de Família e Comunidade, 15 (42).

Blackhall, K. K., Downie, I. P., Ramchandani, P., Shields, H., Brennan, P. A., \& Singh, R. P. (2020). Provision of Emergency Maxillofacial Service During the COVID-19 Pandemic: A Collaborative Five Centre UK Study. The British journal of oral \& maxillofacial surgery, 58(6), 698-703. https://doi.org/10.1016/j.bjoms.2020.05.020

Cabral, E. R., Bonfada, D., de Melo, M. C., Cesar, I. D., de Oliveira, R. E. M., Bastos, T. F., \& Zago, A. C. W. (2020). Contribuições e desafios da Atenção Primária à Saúde frente à pandemia de COVID-19. Interamerican Journal of medicine and health, 3, 1-12. https://doi.org/10.31005/iajmh.v3i0.87

Carvalho, G. C. F., Elias, L. M., \& Carvalho, R. T. (2020). Você sabe o que é uma UTI e sua importância na COVID-19? Secretária de Estado de Minas Gerais. http://coronavirus.saude.mg.gov.br/blog/61-o-que-e-uma-uti.

Cervino, G., \& Oteri, G. (2020). COVID-19 Pandemic and Telephone Triage before Attending Medical Office: Problem or Oportunity?. Medicina (Kaunas, Lithuania), 56(5), 250. https://doi.org/10.3390/medicina56050250

Dann, L., Fitzsimons, J., Gorman, K. M., Hourihame, J. \& Okafor, I. (2020). Disappearing act: COVID-19 and paediatric emergency department attendances. Archives of disease in childhood, 105(8), 810-811. https://doi.org/10.1136/archdischild-2020-319654

Engstrom, E., Melo, E. Giovanella, L., Mendes, A., Grabois., \& de Mendonça, M. H. M. (2020). Recomendações para organização da atenção primaria a saúde no SUS no enfrentamento da COVID-19. Observatório COVID-19, Fiocruz. https://portal.fiocruz.br/documento/recomendacoes-para-organizacao-daaps-no-sus-no-enfrentamento-da-covid-19

Farias, L. A. B. G., Colares, M. P., Barreto, F. K. A., \& Calvacanti, L. P. G. (2020) O Papel Da Atenção Primária No Combate Ao Covid-19: Impacto Na Saúde Pública E Perspectivas Futuras, Revista Brasileira De Medicina E Farmácia, 15 (42). Https://Doi.Org/10.5712/Rbmfc15(42)2455

Filho Silva, P. S. P., Silva e Reis, R. L., Paiva de, M. L. R., da Silva, A. N., dos Santos, B. S. P., de Sou sa, D. C., Moreira, G. B. O., Brito, A. C. S., da Silva, F. J. A., Leal, E. L. G., da Silva, T. S. M., de Araújo, R. M., Figueiredo, B. L. S., Nascimento, H. M. S., \& Cunha, B. P. R. A Importância da Atenção Primária à Saúde Em Pacientes Acometidos Pela Covid-19. Research, Society and Development, 9 (10). http://dx.doi.org/10.33448/rsd-v9i10.8260

Gaythorpe, K., Green, W., Hamlet, A., Hinsley, W., Okell, L. C., Elsland, S. V., Thompson, H., Verity, R., Volz, E., Wang, H., Wang, Y., Walker, P. G. T., Walters, C., Winskill, P., Whittaker, C., Donnelly, C.A., Riley, S., \& Ghani. A. C. (2020) Impact of non-pharmaceutical interventions (NPIs) to reduce COVID-19 mortality and healthcare demand. Imperial College COVID-19 Response Team. 4-20. https://doi.org/10.25561/77482 
Huang, C., Wang, Y., Li, X., Ren, L., Zhao, J., \& Hu, Y. (2020). Clinical features of patients infected with 2019 novel coron avirus in Wuhan, China. The Lancet, 395(10223), 497-506. https://www.thelancet.com/journals/ lancet/article/PIIS0140-6736(20)30183-5/fulltext

Hughes, H. E., Hughes, T. C., Morbey, R., Challen, K., Oliver, K., Smith, G. E., \& Elliot, A. J. (2020). Emergency department use during COVID-19 as described by syndromic surveillance. Emergency medicine journal: EMJ, 37(10), 600-604. https://doi.org/10.1136/emermed-2020-209980

Iser, B. P. M., Silva, I., Raymundo, V. T., Poleto, M. B., Schuelter-Trevisol, F., \& Bobinski, F. (2020). Suspected COVID-19 case definition: a narrative review of the most frequent signs and symptoms among confirmed cases. Definição de caso suspeito da COVID-19: uma revisão narrativa dos sinais e sintomas mais frequentes entre os casos confirmados. Epidemiologia e servicos de saúde. Revista do Sistema Unico de Saude do Brasil, 29(3).

Leow, S. H., Dean, W., MacDonald-Nethercott, M., MacDonald-Nethercott, E., \& Boyle, A. A. (2020). The Attend Study: A Retrospective Observational Study of Emergency Department Attendances During the Early Stages of the COVID-19 Pandemic. Cureus, 12(7). https://doi.org/10.7759/cureus.9328

Lerner, E. B., Newgard, C. D., \& Mann, C. (2020). Effect of the Coronavirus Disease 2019 (COVID-19) Pandemic on the U.S. Emergency Medical Services System: A Preliminary Report. Academic emergency medicine: official journal of the Society for Academic Emergency Medicine,27(8), 693-699. https://doi.org/10.1111/acem.14051

Lin, C. H., Tseng, W. P., W, J. L., Tay, J., Cheng, M. T., Ong, H. N., Lin, H. Y., Chen, Y. Y., Wu, C. H., Chen, J. W., Chen, S. Y., Chan, C. C., Huang, C. H., \& Chen, S. C. (2020). A Double Triage and Telemedicine Protocol to Optimize Infection Control in an Emergency Department in Taiwan During the COVID19 Pandemic: Retrospective Feasibility Study. Journal of medical Internet research, 22(6). https://doi.org/10.2196/20586

Lourenção, L. G. (2020) A COVID-19 e os desafios para o sistema e os profissionais de saúde. Revista enfermagem em foco, 11(1), 2-3.

Low, T. Y., Mathews, I., Liang-Lau, J. W., \& Ngiam, K. Y. (2020). Close air support: enhancing emergency care in the COVID-19 pandemic. Emergency medicine journal: EMJ, 37(10), 642-643. https://doi.org/10.1136/emermed-2020-210148

Mazon, L. M., Komuchena, K. S., Roik, A. K., Wieczorkievicz, A. M., \& Ditterich, R. G. Perfil epidemiológico de pacientes com síndrome gripal e síndrome respiratória aguda grave. Saúde Revista, 16 (43), 37-44.

McDonnell, T. McAuliffe, E., Barrett, M., Cronlon, C., Cummins, F., Deasy, C., Hensey, C., Martin, C., \& Nicholson, E. (2020). CUPID COVID-19: emergency department attendance by paediatric patients during COVID-19 - project protocol. HRB open research, 3 (37). https://doi.org/10.12688/hrbopenres.13066.2

McDonnell, T., Nicholson, E., Conlon, C., Barrett., Cummins, F., Hensey, C., \& McAuliffe, E. (2020). Assessing the Impact of COVID-19 Public Health Stages on Paediatric Emergency Attendance. International journal of environmental research and public health, 17(18). https://doi.org/10.3390/ijerph17186719

Ministério da Saúde (BR). Projeto Lean nas Emergências. (2020). https://portalarquivos.saude.gov.br/images/pdf/2020/April/03/Ebook-SirioLibanesPlanodeCriseCOVID-19-LeannasEmerg--ncias-0304-espelhadas.pdf

Ministério da Saúde (BR). Secretaria de Atenção Especializada à Saúde, Departamento de Atenção Hospitalar, Domiciliar e de Urgência. (2020). Protocolo de Manejo Clínico para o Novo Coronavirus (2019-nCoV). https://www.saude.gov.br/images/pdf/2020/fevereiro/11/protocolo-manejo-coronavirus.pdf

Ministério da Saúde. Centro de Operações de Emergência em Saúde Pública (2020). Boletim COE COVID-19 no. 13: Situação epidemiológica--Doença pelo coronavírus 2019. https:// portalarquivos.saude.gov.br/images/pdf/2020/April/21/BE13--- Boletim-do-COE.pdf

Mitchell, R. D., Reilly, G. M. O., Mitra, B., Smit, D. V., Miller, J. P., \& Cameron, P. A. (2020). Impact of COVID-19 State of Emergency restrictions on presentations to two Victorian emergency departments. Emergency medicine Australasia: EMA, 32(6), 1027-1033. https://doi.org/10.1111/1742-6723.13606

Mulholland, R. H., Wood, R., \& Stagg, H. R. (2020). Impact of COVID-19 on accident and emergency attendances and emergency and planned hospital admissions in Scotland: an interrupted time-series analysis. Journal of the Royal Society of Medicine, 113(11), 444-453. https://doi.org/10.1177/0141076820962447

O'Leary, F., Pobre, K., Mariano, M., Tan, K. F., \& Jani, S. (2020). Personal protective equipment in the paediatric emergency department during the COVID19 pandemic: Estimating requirements based on staff numbers and patient presentations. Emergency medicine Australasia: EMA, 32(6), $1046-1051$. https://doi.org/10.1111/1742-6723.13653

Santana, R., Sousa, J. S., Soares, P. L., Boto, P., \& Rocha, J. V. (2020). The Demand for Hospital Emergency Services: Trends during the First Month of COVID-19 Response. Portuguese Journal of Public Health, 38(1), 23-29. https://dx.doi.org/10.1159/000507764

Schrading, W. A., Pigot, D., \& Tompson, L. (2020). Virtual Remote Attending Supervision in an Academic Emergency Department During the COVID-19 Pandemic. AEM education and training, 4(3), 266-269. https://doi.org/10.1002/aet2.10460

Schuchmann, A. Z., Schnorrenberger, B. L., Chiquetti, M. E., Gaiki, R. S., Raimann, B. W., \& Maeyama, M. A. (2020). Dilemas sanitários e sociais no enfrentamento da pandemia de COVID-19 / Isolamento social vertical X Isolamento social horizontal: saúde e dilemas sociais no enfrentamento da pandemia COVID-19. Revista Brasileira de Revisão de Saúde, 3 (2). https://doi.org/10.34119/bjhrv3n2-185

Silva, L. L. S., Lima, A. F. R., Polli, D. A., Razia, P. F. S., Pavão, L. F. A., Cavalcanti, M. A. F. H., \& Toscano, C. M. (2020). Medidas de distanciamento social para o enfrentamento da COVID-19 no Brasil: caracterização e análise epidemiológica por estado. Cadernos de Saúde Pública, 36 (9).

Souza, M. T., Silva, M. D. da., \& Carvalho, R. de. (2010). Revisão integrativa: o que é e como fazer. Einstein, 8(1). https://www.scielo.br/pdf/eins/v8n1/pt_1679-4508-eins-8-1-0102

Wang, J., Zong, L., Zhang, J., Han, S., Walline, J. H., Sun, P., Xu, S., Li, Y., Wang, C., Liu, J., Li, F., Xu, J., Li, Y., Yu, X., \& Zhi, H. (2020). Identifying the effects of an upgraded 'fever clinic' on COVID-19 control and the workload of emergency department: retrospective study in a tertiary hospital in China. BMJ open, 10(8). https://doi.org/10.1136/bmjopen-2020-039177 
Research, Society and Development, v. 10, n. 3, e9110313027, 2021

(CC BY 4.0) | ISSN 2525-3409 | DOI: http://dx.doi.org/10.33448/rsd-v10i3.13027

World Health Organization Geneva: World Health Organization. (2020). Coronavirus disease 2019 (COVID-19): Situation Report - 51. https://www.who. int/docs/default-source/coronaviruse/situation-reports/20200311- sitrep-51-covid-19.pdf?sfvrsn=1ba62e57_10

Yao, W., Wang, T., Jiang, B., Fleisher, L. A., \& Wei, H. (2020). Emergency tracheal intubation in 202 patients with COVID-19 in Wuhan, China: lessons learnt and international expert recommendations. British journal of anaesthesia, 125(1). https://doi.org/10.1016/j.bja.2020.03.026 